\title{
Effect of irrigation salinity on the growth and yield of two Aus rice cultivars of Bangladesh
}

\author{
Tahmina Khanam, Nahid Akhtar*, M.A. Halim and Feroza Hossain \\ Plant Physiology and Biochemistry Laboratory, Department of Botany, \\ Jahangirnagar University, Savar, Dhaka-1342, Bangladesh
}

\begin{abstract}
The experiment was conducted to clarify the growth and yield response of two rice cultivars, BR55 and BR43 under salt stress. Six different concentrations of $\mathrm{NaCl}$ viz 50, 100, 150, 200, 250 and $300 \mathrm{mM}$ and distilled water (control) were applied on the rice cultivars which were grown under pot culture condition. Growth parameters like plant height, tiller number, leaf number and leaf area were negatively affected by salinity in both cultivars. Salt stress caused a significant reduction in yield in both cultivars of rice. Growth reduction was higher in BR43 than in BR55.The reduction in yield and yield parameters were found to be lower in BR55 than those in BR43. The results obtained in the present study suggest that BR55 showed higher salt tolerance than in BR43.
\end{abstract}

Key words: Salinity, growth, yield, cultivars, rice.

\section{INTRODUCTION}

Saline soil is one of the serious abiotic stresses that causes reduced plant growth, development and productivity worldwide (Siringam et al., 2011). Approximately 7\% of the world's total land area, $20 \%$ of the world's cultivated land area and nearly $50 \%$ of the world's irrigated land area are affected by salinity (Zhu, 2001). In Bangladesh, about 1.06 million hectares of arable lands are affected by soil salinity (SRDI, 2010). About one fifth of the total area of Bangladesh is affected by various degrees of salinity (Karim et al., 1982).

Salt stress has a major impact on plant growth and development. The major inhibitory effect of salinity on plant growth and development has been attributed to osmotic inhibition of water availability as well as the toxic effect of salt ions responsible for salinization. In the majority of plants, salt stress leads to changes in gene expression, leading to an increased synthesis of osmoprotectors and osmoregulators (Teixeira \& Pereira, 2007). Salinity imposes two constraints on plants: the hyperosmotic effect (especially short-term stress) due to lower soil water potential, and the hyperionic effect (especially long-term stress) due to direct toxicity of ions over metabolism and nutrition imbalance of plants (Verma \& Mishra, 2005; Duan et al., 2008).

Rice is the staple food for the people of Bangladesh and plays the most important role in the national economy. Bangladesh is the $4^{\text {th }}$ largest country in Asia with respect to rice

\footnotetext{
* Corresponding author: E-mail: nahid_akhtar98@yahoo.com
} 
area and production (BBS, 2004). It occupies $74 \%$ of the total cropped area, accounts for $70 \%$ of the value of crop output and contributes $20 \%$ to GDP (BBS, 2001). It contributes $75.6 \%$ of the calorie, $66 \%$ of the protein and $17.8 \%$ of fat (FAOSTAT, 2001). Per capita cereal consumption is $150.4 \mathrm{~kg}$ of which share of rice is $91 \%$. However, rice production may not achieve its goal due to natural disasters, soil salinity and ambient air pollution in Bangladesh. Thus, it is urgent for the researchers and policy makers to search alternative techniques for better utilization of stress-prone areas, like screening of salt tolerant cultivars might be the best approach to bring salinity prone areas under cultivation and will ensure the country's food security.

There is increasing evidence that salt stress has a significant effect on growth and yield of plants and rated as a salt sensitive crop. Inspite of extensive research or information data available or salinity impact on rice but quantitative effect of salinity on different growth stages, yield components is limited. The present study was aimed to clarify the effect of salinity on growth, development and yield of two contrasting salt tolerance rice cultivars at different stress duration.

\section{MATERIALS AND METHODS}

Plant materials: Two rice cultivars (Oryza sativa L.), BR55 and BR43 were used as plant materials. Rice seeds were collected from Bangladesh Rice Research Institute (BRRI). Both cultivars are Aus rice.

Treatments: Six different levels of $\mathrm{NaCl}$ solution (50, 100, 150, 200, 250 and $300 \mathrm{mM}$ ) and distilled water as control were applied in this experiment.

Pot preparation and management practices: Pot experiments were carried out in the experimental field of Botanical garden of Jahangirnagar University, Savar, Dhaka. Pots were filled with (12 kg per pot) air dried soil. The $\mathrm{P}^{\mathrm{H}}$ value and cation exchange capacity of the soil were 7.12 and $0.20(\mathrm{mg} / 100 \mathrm{~g}$ dry soil) respectively. Before sowing $0.41 \mathrm{gm}$ of TSP, $0.5 \mathrm{gm}$ of MOP and $0.81 \mathrm{gm}$ of gypsum per pot were incorporated into the soil and sufficient water was added to saturate the soil. The pots were kept under natural sunshine till harvesting. Seeds of uniform size were directly sown in pots. Distilled water was applied in all pots up to the emergence of seedlings. After seedling establishment distilled water in control pots and $12.5 \mathrm{mM} \mathrm{NaCl}$ solution were applied in salt treatment. When the first leaf appeared, actual amount of $\mathrm{NaCl}$ solution were applied at 3 days interval. The salt solution were applied till harvest. Regular watering and weeding were made to ensure equal environmental condition throughout the experimental period.

Measurement of growth and yield: Plant height, leaf number, tiller number and leaf area of two rice cultivars were measured at 10, 20, 30 and 40 days after treatment (DAT). Yield and yield contributing parameters like yield per plant, filled grain number per plant, total grain number per plant and panicle number per plant were recorded at final harvest.

Statiscal analysis: The experiment was arranged out in randomized block design with three replications. Statistical analysis of the collected data were performed using Analysis 
of Varience (ANOVA). All statistical analysis were performed with the SPSS statistical package (SPSS program 16.00). Tukey's HSD test was performed to identify significant differences among the treatments.

\section{RESULTS AND DISCUSSION}

\section{Measurement of Growth}

Plant height: Plant height decreased significantly with the increasing levels of $\mathrm{NaCl}$ (Table 1). Compared to control, the highest salinity level significantly reduced plant height by $31.74 \%$ for BR55 and $62.22 \%$ for BR43 at 10 DAT. Compared to control, under $300 \mathrm{mM} \mathrm{NaCl}$ concentration plant height at 20 DAT was reduced by $29.54 \%$ for BR55 and 33.20\% for BR43. At 30 DAT, plant height was reduced $29.18 \%$ for BR55 and $35.60 \%$ for BR43compared to the control under $300 \mathrm{mM} \mathrm{NaCl}$ concentration. Compared to control, the highest salinity level significantly reduced plant height by $30.51 \%$ for BR55 and $35.01 \%$ for BR43 at 40 DAT. Salinity significantly reduced the plant height in both cultivars. However salinity induced reduction of plant height was higher in BR43 than that in BR55.

Inhibition of growth due to salt stress has been observed even in tolerant plant species (Mittler et al., 2001). Shoot and root growth reductions are the most important agricultural indices of salt tolerance (Tuna et al., 2008). Earlier workers have reported reduction of plant height in rice under salt stress (Weon et al., 2003; Islam et al., 2007). The salt induced reduction in plant height could be due to the negative effect of this salt on the rate of photosynthesis, the changes in enzyme activity (that subsequently affects protein synthesis) and also the decrease in the level of carbohydrates and growth hormones (Mazher et al., 2007).

Tiller number: Tiller number also significantly decreased with the increasing levels of $\mathrm{NaCl}$ (Table 2). Compared to control, the highest salinity level significantly reduced tiller number by $56.03 \%$ for BR55 and $69.96 \%$ for BR43 at 10 DAT. Compared to control, under $300 \mathrm{mM} \mathrm{NaCl}$ concentration, tiller number at 20 DAT was reduced by $47.52 \%$ for BR55 and $62.02 \%$ for BR43. At 30 DAT, tiller number was reduced by $43.07 \%$ for BR55 and $57.28 \%$ for BR43compared to the control under $300 \mathrm{mM} \mathrm{NaCl}$ concentration. Compared to control, the highest salinity level significantly reduced tiller number by 43.58\% for BR55 and 54.54\% for BR43at 40 DAT. However, salinity induced reduction of tiller number was higher in BR43 than that in BR55.

Zeng et al. (2003b) reported that salinity decreases number of tillers while imposing before panicle emergence. Eugene et al. (1994) reported that salinity stress strongly influenced the distribution of spike-bearing tillers. Nicolas et al. (1994) found that salt stress during tiller emergence can inhibit their formation and can cause their abortion at later stages. 
Table 1. Effects of different concentrations of salt $(\mathrm{NaCl})$ on plant height $(\mathrm{cm})$ of two rice cultivars BR55 and BR43 at different days after treatment

\begin{tabular}{|c|c|c|c|c|c|c|c|c|}
\hline \multirow{3}{*}{ Treatments } & \multicolumn{8}{|c|}{ Days after treatment (DAT) } \\
\hline & \multicolumn{2}{|c|}{10} & \multicolumn{2}{|c|}{20} & \multicolumn{2}{|c|}{30} & \multicolumn{2}{|c|}{40} \\
\hline & BR55 & BR43 & BR55 & BR43 & BR55 & BR43 & BR55 & BR43 \\
\hline $0 \mathrm{mM}$ & $70.16 \pm 2.17 \mathrm{a}$ & $65.44 \pm 1.56 \mathrm{a}$ & $82.53 \pm 1.79 a$ & $77.65 \pm 1.77 a$ & $93.52 \pm 2.35 \mathrm{a}$ & $86.45 \pm 1.63 \mathrm{a}$ & $102.38 \pm 2.27 \mathrm{a}$ & $95.07 \pm 1.62 \mathrm{a}$ \\
\hline $50 \mathrm{mM}$ & $\begin{array}{c}67.56 \pm 1.87 \mathrm{a} \\
(96.29)\end{array}$ & $\begin{array}{c}59.32 \pm 1.78 b \\
(90.64)\end{array}$ & $\begin{array}{c}78.07 \pm 1.86 \mathrm{~b} \\
(94.59)\end{array}$ & $\begin{array}{c}72.93 \pm 2.59 b \\
(93.92)\end{array}$ & $\begin{array}{c}89.34 \pm 1.53 \mathrm{a} \\
(95.53)\end{array}$ & $\begin{array}{c}81.34 \pm 1.93 \mathrm{a} \\
(94.08)\end{array}$ & $\begin{array}{c}97.26 \pm 2.74 \mathrm{~b} \\
(94.99)\end{array}$ & $\begin{array}{c}91.03 \pm 2.22 b \\
(95.75)\end{array}$ \\
\hline $100 \mathrm{mM}$ & $\begin{array}{c}62.76 \pm 2.12 b \\
(89.45)\end{array}$ & $\begin{array}{c}52.67 \pm 2.65 c \\
(80.48)\end{array}$ & $\begin{array}{c}73.03 \pm 1.69 \mathrm{c} \\
(88.48)\end{array}$ & $\begin{array}{c}67.9 \pm 2.38 \mathrm{c} \\
(87.44)\end{array}$ & $\begin{array}{c}82.76 \pm 1.43 b \\
(88.49)\end{array}$ & $\begin{array}{c}73.54 \pm 2.03 b \\
(85.06)\end{array}$ & $\begin{array}{c}91.04 \pm 2.70 \mathrm{c} \\
(88.92)\end{array}$ & $\begin{array}{c}84.56 \pm 1.93 \mathrm{c} \\
(88.94)\end{array}$ \\
\hline $150 \mathrm{mM}$ & $\begin{array}{c}58.88 \pm 2.74 \mathrm{c} \\
(83.92)\end{array}$ & $\begin{array}{c}43.24 \pm 1.89 \mathrm{~d} \\
(66.07)\end{array}$ & $\begin{array}{c}68.02 \pm 2.84 \mathrm{~d} \\
(82.41)\end{array}$ & $\begin{array}{c}62.83 \pm 2.32 \mathrm{~d} \\
(80.91)\end{array}$ & $\begin{array}{c}77.34 \pm 2.10 \mathrm{c} \\
(82.69)\end{array}$ & $\begin{array}{c}67.44 \pm 1.56 \mathrm{c} \\
(78.01)\end{array}$ & $\begin{array}{c}86.24 \pm 2.19 \mathrm{~d} \\
(84.23)\end{array}$ & $\begin{array}{c}79.14 \pm 2.50 \mathrm{~d} \\
(83.24)\end{array}$ \\
\hline $200 \mathrm{mM}$ & $\begin{array}{c}53.23 \pm 1.45 \mathrm{~d} \\
(75.86)\end{array}$ & $\begin{array}{c}36.45 \pm 2.34 \mathrm{e} \\
(55.69)\end{array}$ & $\begin{array}{c}64.08 \pm 2.74 \mathrm{e} \\
(77.64)\end{array}$ & $\begin{array}{c}59.26 \pm 2.85 \mathrm{~d} \\
(76.31)\end{array}$ & $\begin{array}{c}73.23 \pm 1.67 \mathrm{~cd} \\
(78.30)\end{array}$ & $\begin{array}{c}62.96 \pm 1.88 \mathrm{~cd} \\
(72.82)\end{array}$ & $\begin{array}{c}80.17 \pm 2.75 \mathrm{e} \\
(78.30)\end{array}$ & $\begin{array}{c}72.83 \pm 2.87 \mathrm{e} \\
(76.60)\end{array}$ \\
\hline $250 \mathrm{mM}$ & $\begin{array}{c}50.47 \pm 2.23 \mathrm{de} \\
(71.93)\end{array}$ & $\begin{array}{c}30.76 \pm 2.56 f \\
(47.00)\end{array}$ & $\begin{array}{c}61.11 \pm 2.57 \mathrm{ef} \\
(74.04)\end{array}$ & $\begin{array}{c}55.25 \pm 2.55 \mathrm{e} \\
(71.15)\end{array}$ & $\begin{array}{c}68.56 \pm 1.96 \mathrm{de} \\
(73.31)\end{array}$ & $\begin{array}{c}58.56 \pm 2.31 \mathrm{de} \\
(67.73)\end{array}$ & $\begin{array}{c}76.26 \pm 2.81 \mathrm{f} \\
(74.48)\end{array}$ & $\begin{array}{c}67.26 \pm 2.54 \mathrm{f} \\
(70.74)\end{array}$ \\
\hline $300 \mathrm{mM}$ & $\begin{array}{c}47.89 \pm 1.42 \mathrm{e} \\
(68.25)\end{array}$ & $\begin{array}{c}24.72 \pm 1.55 \mathrm{~g} \\
(37.77)\end{array}$ & $\begin{array}{c}58.15 \pm 2.04 \mathrm{f} \\
(70.45)\end{array}$ & $\begin{array}{c}51.87 \pm 2.69 \mathrm{e} \\
(66.79)\end{array}$ & $\begin{array}{c}66.23 \pm 1.18 \mathrm{e} \\
(70.81)\end{array}$ & $\begin{array}{c}55.67 \pm 1.45 \mathrm{e} \\
(64.39)\end{array}$ & $\begin{array}{c}71.14 \pm 2.73 \mathrm{~g} \\
(69.48)\end{array}$ & $\begin{array}{c}61.78 \pm 2.96 \mathrm{~g} \\
(64.98)\end{array}$ \\
\hline $\begin{array}{l}\text { Cultivar } \\
\text { Mean }\end{array}$ & 58.7 & 44.65 & 69.28 & 63.95 & 78.71 & 69.42 & 86.35 & 78.81 \\
\hline $\mathrm{CV} \%$ & 13.98 & 32.23 & 12.51 & 14.18 & 12.59 & 15.88 & 12.57 & 14.84 \\
\hline
\end{tabular}

* Average value of 9 plants in each treatment.

* Means in a column followed by the same letter do not differ significantly at $5 \%$ level and \pm means standard deviation.

* Values within parenthesis indicate percentage relative to the control. 
Irrigation salinity, growth and yield, Aus rice cultivars, Bangladesh

Table 2. Effects of different concentrations of salt ( $\mathrm{NaCl})$ on tiller number of two rice cultivars BR55 and BR43 at different days after treatment

\begin{tabular}{|c|c|c|c|c|c|c|c|c|}
\hline \multirow{3}{*}{ Treatments } & \multicolumn{8}{|c|}{ Days after treatment (DAT) } \\
\hline & \multicolumn{2}{|c|}{10} & \multicolumn{2}{|c|}{20} & \multicolumn{2}{|c|}{30} & \multicolumn{2}{|c|}{40} \\
\hline & BR55 & BR43 & BR55 & BR43 & BR55 & BR43 & BR55 & BR43 \\
\hline $0 \mathrm{mM}$ & $6.55 \pm 0.56 a$ & $6.66 \pm 0.63 \mathrm{a}$ & $8.88 \pm 0.78 a$ & $8.77 \pm 0.83 a$ & $10.33 \pm 1.20 \mathrm{a}$ & $9.88 \pm 1.02 \mathrm{a}$ & $12.00 \pm 1.00 \mathrm{a}$ & $11.00 \pm 1.00 \mathrm{a}$ \\
\hline $50 \mathrm{mM}$ & $\begin{array}{c}5.88 \pm 0.73 \mathrm{ab} \\
(89.77)\end{array}$ & $\begin{array}{c}5.33 \pm 1.00 \mathrm{ab} \\
(80.03)\end{array}$ & $\begin{array}{c}8.11 \pm 0.92 \mathrm{ab} \\
(91.32)\end{array}$ & $\begin{array}{c}7.44 \pm 1.01 \mathrm{ab} \\
(84.83)\end{array}$ & $\begin{array}{c}9.88 \pm 0.47 \mathrm{a} \\
(95.64)\end{array}$ & $\begin{array}{c}9.11 \pm 0.98 \mathrm{ab} \\
(92.02)\end{array}$ & $\begin{array}{c}11.22 \pm 1.39 \mathrm{ab} \\
(93.50)\end{array}$ & $\begin{array}{c}10.11 \pm 1.36 \mathrm{ab} \\
(91.90)\end{array}$ \\
\hline $100 \mathrm{mM}$ & $\begin{array}{c}5.11 \pm 1.03 \mathrm{ab} \\
(78.01)\end{array}$ & $\begin{array}{c}4.22 \pm 0.43 \mathrm{abc} \\
(63.36)\end{array}$ & $\begin{array}{c}7.55 \pm 0.88 b c \\
(85.02)\end{array}$ & $\begin{array}{c}6.11 \pm 0.92 b c \\
(69.66)\end{array}$ & $\begin{array}{c}9.11 \pm 0.78 \mathrm{ab} \\
(88.18)\end{array}$ & $\begin{array}{c}8.33 \pm 0.65 a b c \\
(84.31)\end{array}$ & $\begin{array}{c}10.44 \pm 0.88 \mathrm{bc} \\
(87.00)\end{array}$ & $\begin{array}{c}9.22 \pm 1.09 \mathrm{bc} \\
(83.81)\end{array}$ \\
\hline $150 \mathrm{mM}$ & $\begin{array}{c}4.88 \pm 0.24 \mathrm{ab} \\
(74.50)\end{array}$ & $\begin{array}{c}3.88 \pm 0.78 \mathrm{abc} \\
(58.25)\end{array}$ & $\begin{array}{c}6.77 \pm 0.66 \mathrm{~cd} \\
(76.23)\end{array}$ & $\begin{array}{c}5.44 \pm 0.88 \mathrm{c} \\
(62.02)\end{array}$ & $\begin{array}{c}8.66 \pm 1.17 \mathrm{abc} \\
(83.83)\end{array}$ & $\begin{array}{c}6.88 \pm 1.08 \mathrm{bcd} \\
(69.63)\end{array}$ & $\begin{array}{c}9.66 \pm 1.00 \mathrm{~cd} \\
(80.50)\end{array}$ & $\begin{array}{c}8.00 \pm 1.22 \mathrm{~cd} \\
(72.72)\end{array}$ \\
\hline $200 \mathrm{mM}$ & $\begin{array}{c}3.99 \pm 0.92 \mathrm{ab} \\
(60.91)\end{array}$ & $\begin{array}{c}3.11 \pm 1.26 \mathrm{bc} \\
(46.69)\end{array}$ & $\begin{array}{c}5.77 \pm 0.66 \mathrm{de} \\
(64.97)\end{array}$ & $\begin{array}{c}5.00 \pm 1.32 \mathrm{~cd} \\
(57.01)\end{array}$ & $\begin{array}{c}7.00 \pm 0.75 b c \\
(67.76)\end{array}$ & $\begin{array}{c}6.11 \pm 0.48 \mathrm{~cd} \\
(61.84)\end{array}$ & $\begin{array}{c}8.88 \pm 0.92 \mathrm{de} \\
(74.00)\end{array}$ & $\begin{array}{c}6.77 \pm 0.83 \mathrm{de} \\
(61.54)\end{array}$ \\
\hline $250 \mathrm{mM}$ & $\begin{array}{c}3.33 \pm 0.61 b \\
(50.83)\end{array}$ & $\begin{array}{c}2.77 \pm 0.56 \mathrm{bc} \\
(41.59)\end{array}$ & $\begin{array}{c}5.00 \pm 0.70 \mathrm{e} \\
(56.30)\end{array}$ & $\begin{array}{c}4.00 \pm 0.70 \mathrm{de} \\
(45.61)\end{array}$ & $\begin{array}{c}6.33 \pm 0.94 b c \\
(61.27)\end{array}$ & $\begin{array}{c}5.55 \pm 1.00 \mathrm{~cd} \\
(56.17)\end{array}$ & $\begin{array}{c}7.88 \pm 1.05 \mathrm{ef} \\
(65.66)\end{array}$ & $\begin{array}{c}5.88 \pm 0.78 \mathrm{ef} \\
(53.45)\end{array}$ \\
\hline $300 \mathrm{mM}$ & $\begin{array}{c}2.88 \pm 0.84 \mathrm{~b} \\
(43.96)\end{array}$ & $\begin{array}{c}2.00 \pm 0.54 \mathrm{c} \\
(30.03)\end{array}$ & $\begin{array}{c}4.66 \pm 0.86 \mathrm{e} \\
(52.47)\end{array}$ & $\begin{array}{c}3.33 \pm 0.70 \mathrm{e} \\
(37.97)\end{array}$ & $\begin{array}{c}5.88 \pm 0.62 \mathrm{c} \\
(56.92)\end{array}$ & $\begin{array}{c}4.22 \pm 0.73 \mathrm{~d} \\
(42.71)\end{array}$ & $\begin{array}{c}6.77 \pm 0.83 \mathrm{f} \\
(56.41)\end{array}$ & $\begin{array}{c}5.00 \pm 1.11 \mathrm{f} \\
(45.45)\end{array}$ \\
\hline $\begin{array}{c}\text { Cultivar } \\
\text { Mean }\end{array}$ & 4.66 & 3.99 & 6.67 & 5.72 & 8.17 & 7.15 & 9.55 & 7.99 \\
\hline $\mathrm{CV} \%$ & 33.5 & 43.21 & 25.04 & 34.64 & 22.92 & 29.47 & 20.74 & 29.01 \\
\hline
\end{tabular}

* Average value of 9 plants in each treatment

* Means in a column followed by the same letter do not differ significantly at $5 \%$ level and \pm means standard deviation.

* Values within parenthesis indicate percentage relative to the control. 
Leaf number: Salinity disturbed seriously the production of leaf, which was depicted in the stiff reduction in leaf number/plant (Table 3).Compared to control, the highest salinity level significantly reduced leaf number by $56.46 \%$ for BR55 and $60.94 \%$ for BR43 at 10 DAT. Compared to control, under $300 \mathrm{mM} \mathrm{NaCl}$ concentration leaf number at 20 DAT was reduced by $49.23 \%$ for BR55 and $58.03 \%$ for BR43. At 30 DAT, leaf number was reduced $45.82 \%$ for BR55 and $58.22 \%$ for BR43compared to the control under $300 \mathrm{mM}$ $\mathrm{NaCl}$ concentration. Compared to control, the highest salinity level significantly reduced leaf number by $44.67 \%$ for BR55 and $57.21 \%$ for BR43 at 40 DAT. However, under salinity stress reduction of leaf number was higher in BR43 than that in BR55.

Green leaves and dry matter production per plant were reported to be reduced with the increase in soil salinity (Bal \& Dutt, 1984). Inhibition of the formation of leaf primordia under salt stress could be the probable reason for low leaf number (Alamgir \& Ali, 2006). The decrease of leaf numbers may be due to the accumulation of sodium chloride in the cell walls and cytoplasm of the older leaves.

Leaf area: Salinity significantly decreased the production of green leaf area (Table 4).Compared to control, the highest salinity level significantly reduced leaf area by $51.03 \%$ for BR55 and $60.34 \%$ for BR43 at 10 DAT. Compared to control, under $300 \mathrm{mM}$ $\mathrm{NaCl}$ concentration leaf area at 20 DAT was reduced by $45.18 \%$ for BR55 and $55.36 \%$ for BR43. At 30 DAT, leaf area was reduced $42.36 \%$ for BR55 and $56.36 \%$ for BR43compared to the control under $300 \mathrm{mM} \mathrm{NaCl}$ concentration. Compared to control, the highest salinity level significantly reduced leaf area by $42.09 \%$ for BR55 and 55.23\% for BR43 at 40 DAT. However, reduction in leaf area at different salinity level were higher in BR43 than that in BR55.

Salinity has been reported to decrease leaf area tremendously (Wankhade et al., 2013). The decrease in leaf area, found in this study could be explained by the negative effect of salt on photosynthesis that leads to the reduction of plant growth and leaf growth (Netondo et al., 2004).

\section{Measurement of Yield}

Yield number per plant (filled grain weight): Salinity significantly decreased the grain yield (Table 5). At all levels of salinity the absolute grain yield of BR55 was significantly higher than BR43.Compared to control, the highest salinity level significantly reduced yield number per plant by $67.1 \%$ for BR55 and $76.68 \%$ for BR 43 . Salinity induced significant higher reduction of grain yield was observed in BR43 than that in BR55. Thus, this results summarized that BR43 is highly sensitive to salt stress than BR55.

Reduction in yield due to salt stress has been reported by Zeng \& Shannon (2000a) and Cha-um \& Kirdmanee (2010). Rice yield is often decreased with increasing salinity especially when experienced in the early development stages (Menete et al., 2008). Salinity affected the grain yield through a reduction in various components such as spike number and grain number in most of the genotypes (Saqib et al., 2012). 
Table 3. Effects of different concentrations of salt ( $\mathrm{NaCl})$ on leaf number of two rice cultivars BR55 and BR43 at different days after treatment

\begin{tabular}{|c|c|c|c|c|c|c|c|c|}
\hline \multirow{3}{*}{ Treatments } & \multicolumn{8}{|c|}{ Days after treatment (DAT) } \\
\hline & \multicolumn{2}{|c|}{10} & \multicolumn{2}{|c|}{20} & \multicolumn{2}{|c|}{30} & \multicolumn{2}{|c|}{40} \\
\hline & BR55 & BR43 & BR55 & BR43 & BR55 & BR43 & BR55 & BR43 \\
\hline $0 \mathrm{mM}$ & $29.33 \pm 1.73 a$ & $24.43 \pm 2.57 \mathrm{a}$ & $36.11 \pm 2.08 \mathrm{a}$ & $32.55 \pm 2.65 \mathrm{a}$ & $42.66 \pm 1.23 \mathrm{a}$ & $39.62 \pm 1.56 a$ & $49.00 \pm 2.00 \mathrm{a}$ & $47.00 \pm 3.39 \mathrm{a}$ \\
\hline $50 \mathrm{mM}$ & $\begin{array}{c}25.11 \pm 2.10 \mathrm{ab} \\
(85.61)\end{array}$ & $\begin{array}{c}21.67 \pm 2.89 \mathrm{ab} \\
(88.70)\end{array}$ & $\begin{array}{c}32.11 \pm 1.90 b \\
(88.92)\end{array}$ & $\begin{array}{c}29.55 \pm 3.00 \mathrm{a} \\
(90.78)\end{array}$ & $\begin{array}{c}38.44 \pm 1.67 \mathrm{ab} \\
(90.10)\end{array}$ & $\begin{array}{c}35.86 \pm 1.96 \mathrm{ab} \\
(90.50)\end{array}$ & $\begin{array}{c}44.88 \pm 2.31 \mathrm{~b} \\
(91.59)\end{array}$ & $\begin{array}{c}40.33 \pm 3.27 b \\
(85.80)\end{array}$ \\
\hline $100 \mathrm{mM}$ & $\begin{array}{c}22.55 \pm 2.54 \mathrm{bc} \\
(76.88)\end{array}$ & $\begin{array}{c}19.34 \pm 1.67 \mathrm{abc} \\
(79.16)\end{array}$ & $\begin{array}{c}30 \pm 1.22 \mathrm{~b} \\
(83.07)\end{array}$ & $\begin{array}{c}24.22 \pm 2.58 \mathrm{~b} \\
(74.40)\end{array}$ & $\begin{array}{c}36.22 \pm 2.19 b \\
(84.90)\end{array}$ & $\begin{array}{c}30.43 \pm 2.18 \mathrm{bc} \\
(76.80)\end{array}$ & $\begin{array}{c}41.77 \pm 0.88 \mathrm{bc} \\
(85.24)\end{array}$ & $\begin{array}{c}36.33 \pm 3.27 \mathrm{~b} \\
(77.29)\end{array}$ \\
\hline $150 \mathrm{mM}$ & $\begin{array}{c}19.11 \pm 1.82 \mathrm{~cd} \\
(65.15)\end{array}$ & $\begin{array}{c}16.59 \pm 2.48 \mathrm{bc} \\
(67.90)\end{array}$ & $\begin{array}{c}27.11 \pm 2.31 \mathrm{c} \\
(75.07)\end{array}$ & $\begin{array}{c}21.44 \pm 2.78 \mathrm{bc} \\
(65.85)\end{array}$ & $\begin{array}{c}34.11 \pm 1.56 \mathrm{bc} \\
(79.95)\end{array}$ & $\begin{array}{c}26.34 \pm 2.48 \mathrm{~cd} \\
(66.48)\end{array}$ & $\begin{array}{c}38.88 \pm 2.14 \mathrm{~cd} \\
(79.34)\end{array}$ & $\begin{array}{c}31.11 \pm 3.17 \mathrm{c} \\
(66.19)\end{array}$ \\
\hline $200 \mathrm{mM}$ & $\begin{array}{c}17.99 \pm 2.37 \mathrm{cde} \\
(61.33)\end{array}$ & $\begin{array}{c}14.88 \pm 1.74 \mathrm{bcd} \\
(60.90)\end{array}$ & $\begin{array}{c}23.11 \pm 1.53 \mathrm{~d} \\
(63.99)\end{array}$ & $\begin{array}{c}19.33 \pm 2.39 \mathrm{~cd} \\
(59.38)\end{array}$ & $\begin{array}{c}29.33 \pm 1.92 \mathrm{~cd} \\
(68.75)\end{array}$ & $\begin{array}{c}22.75 \pm 1.98 \mathrm{de} \\
(57.42)\end{array}$ & $\begin{array}{c}35.88 \pm 1.90 \mathrm{~d} \\
(73.22)\end{array}$ & $\begin{array}{c}27.22 \pm 2.43 \mathrm{~cd} \\
(57.91)\end{array}$ \\
\hline $250 \mathrm{mM}$ & $\begin{array}{c}14.44 \pm 1.35 \mathrm{de} \\
(49.23)\end{array}$ & $\begin{array}{c}12.56 \pm 2.67 \mathrm{~cd} \\
(51.41)\end{array}$ & $\begin{array}{c}20.11 \pm 2.20 \mathrm{e} \\
(55.69)\end{array}$ & $\begin{array}{c}16.00 \pm 2.59 \mathrm{de} \\
(49.15)\end{array}$ & $\begin{array}{c}26.22 \pm 1.72 \mathrm{de} \\
(61.46)\end{array}$ & $\begin{array}{c}18.78 \pm 1.65 \mathrm{ef} \\
(47.40)\end{array}$ & $\begin{array}{c}31.11 \pm 2.26 \mathrm{e} \\
(63.48)\end{array}$ & $\begin{array}{c}23.44 \pm 3.08 \mathrm{de} \\
(49.87)\end{array}$ \\
\hline $300 \mathrm{mM}$ & $\begin{array}{c}12.77 \pm 2.42 \mathrm{e} \\
(43.53)\end{array}$ & $\begin{array}{c}9.54 \pm 1.47 \mathrm{~d} \\
(39.05)\end{array}$ & $\begin{array}{c}18.33 \pm 2.17 \mathrm{e} \\
(50.76)\end{array}$ & $\begin{array}{c}13.66 \pm 2.12 \mathrm{e} \\
(41.96)\end{array}$ & $\begin{array}{c}23.11 \pm 1.20 \mathrm{e} \\
(54.17)\end{array}$ & $\begin{array}{c}16.55 \pm 1.47 \mathrm{f} \\
(41.77)\end{array}$ & $\begin{array}{c}27.11 \pm 2.14 \mathrm{f} \\
(55.32)\end{array}$ & $\begin{array}{c}20.11 \pm 3.10 \mathrm{e} \\
(42.78)\end{array}$ \\
\hline $\begin{array}{c}\text { Cultivar } \\
\text { Mean }\end{array}$ & 20.18 & 17 & 26.69 & 22.39 & 32.87 & 27.19 & 38.37 & 32.22 \\
\hline $\mathrm{CV} \%$ & 28.72 & 31.49 & 23.83 & 30.78 & 20.75 & 30.65 & 19.4 & 29.24 \\
\hline
\end{tabular}

* Average value of 9 plants in each treatment

* Means in a column followed by the same letter do not differ significantly at $5 \%$ level and \pm means standard deviation.

* Values within parenthesis indicate percentage relative to the control. 
Table 4. Effects of different concentrations of salt $(\mathrm{NaCl})$ on leaf area $\left(\mathrm{cm}^{2}\right) /$ plant of two rice cultivars BR55 and BR43 at different days after Treatment

\begin{tabular}{|c|c|c|c|c|c|c|c|c|}
\hline \multirow{3}{*}{ Treatments } & \multicolumn{8}{|c|}{ Days after treatment (DAT) } \\
\hline & \multicolumn{2}{|c|}{10} & \multicolumn{2}{|c|}{20} & \multicolumn{2}{|c|}{30} & \multicolumn{2}{|c|}{40} \\
\hline & BR55 & BR43 & BR55 & BR43 & BR55 & BR43 & BR55 & BR43 \\
\hline $0 \mathrm{mM}$ & $38.31 \pm 2.53 a$ & $38.68 \pm 2.45 a$ & $49.35 \pm 2.31 \mathrm{a}$ & $47.30 \pm 3.41 \mathrm{a}$ & $58.45 \pm 1.78 \mathrm{a}$ & $55.78 \pm 1.67 a$ & $66.51 \pm 2.10 \mathrm{a}$ & $61.12 \pm 2.59 a$ \\
\hline $50 \mathrm{mM}$ & $\begin{array}{c}35.74 \pm 1.64 a b \\
(93.29)\end{array}$ & $\begin{array}{c}33.42 \pm 1.66 \mathrm{ab} \\
(86.40)\end{array}$ & $\begin{array}{c}46.54 \pm 2.66 \mathrm{a} \\
(94.30)\end{array}$ & $\begin{array}{c}42.02 \pm 3.29 b \\
(88.83)\end{array}$ & $\begin{array}{c}55.64 \pm 1.93 \mathrm{a} \\
(95.19)\end{array}$ & $\begin{array}{c}49.56 \pm 1.59 \mathrm{~b} \\
(88.84)\end{array}$ & $\begin{array}{c}62.53 \pm 2.92 \mathrm{~b} \\
(94.01)\end{array}$ & $\begin{array}{c}53.53 \pm 2.55 b \\
(87.58)\end{array}$ \\
\hline $100 \mathrm{mM}$ & $\begin{array}{c}31.83 \pm 2.85 b c \\
(83.08)\end{array}$ & $\begin{array}{c}28.87 \pm 1.27 \mathrm{bc} \\
(74.63)\end{array}$ & $\begin{array}{c}41.25 \pm 2.55 b \\
(83.58)\end{array}$ & $\begin{array}{c}38.02 \pm 2.94 \mathrm{~b} \\
(80.38)\end{array}$ & $\begin{array}{c}49.34 \pm 2.34 \mathrm{~b} \\
(84.41)\end{array}$ & $\begin{array}{c}43.87 \pm 2.32 \mathrm{c} \\
(78.64)\end{array}$ & $\begin{array}{c}55.23 \pm 3.22 \mathrm{c} \\
(83.04)\end{array}$ & $\begin{array}{c}47.62 \pm 2.49 \mathrm{c} \\
(77.91)\end{array}$ \\
\hline $150 \mathrm{mM}$ & $\begin{array}{c}28.43 \pm 2.47 \mathrm{~cd} \\
(74.21)\end{array}$ & $\begin{array}{c}23.45 \pm 2.80 \mathrm{~cd} \\
(60.62)\end{array}$ & $\begin{array}{c}37.36 \pm 1.70 \mathrm{c} \\
(75.70)\end{array}$ & $\begin{array}{c}33.72 \pm 2.67 \mathrm{c} \\
(71.28)\end{array}$ & $\begin{array}{c}44.71 \pm 2.52 \mathrm{bc} \\
(76.49)\end{array}$ & $\begin{array}{c}38.53 \pm 2.56 \mathrm{~d} \\
(69.07)\end{array}$ & $\begin{array}{l}49.96 \pm 2.25 \mathrm{~d} \\
(75.11)\end{array}$ & $\begin{array}{c}41.66 \pm 3.00 \mathrm{~d} \\
(68.16)\end{array}$ \\
\hline $200 \mathrm{mM}$ & $\begin{array}{c}24.81 \pm 1.56 \mathrm{de} \\
(64.76)\end{array}$ & $\begin{array}{c}20.43 \pm 2.74 \mathrm{de} \\
(52.81)\end{array}$ & $\begin{array}{c}34.44 \pm 2.61 \mathrm{c} \\
(69.78)\end{array}$ & $\begin{array}{c}28.85 \pm 2.93 \mathrm{~d} \\
(60.99)\end{array}$ & $\begin{array}{c}41.53 \pm 1.70 \mathrm{~cd} \\
(71.05)\end{array}$ & $\begin{array}{c}33.74 \pm 2.76 \mathrm{e} \\
(60.48)\end{array}$ & $\begin{array}{c}46.54 \pm 2.55 \mathrm{~d} \\
(69.97)\end{array}$ & $\begin{array}{c}36.47 \pm 1.95 \mathrm{e} \\
(59.66)\end{array}$ \\
\hline $250 \mathrm{mM}$ & $\begin{array}{c}21.72 \pm 2.62 \mathrm{ef} \\
(56.69)\end{array}$ & $\begin{array}{c}17.64 \pm 2.59 \mathrm{e} \\
(45.60)\end{array}$ & $\begin{array}{c}30.07 \pm 1.98 \mathrm{~d} \\
(60.93)\end{array}$ & $\begin{array}{c}24.61 \pm 2.40 \mathrm{e} \\
(52.02)\end{array}$ & $\begin{array}{c}36.57 \pm 2.38 \mathrm{de} \\
(62.56)\end{array}$ & $\begin{array}{c}29.98 \pm 1.78 \mathrm{e} \\
(53.74)\end{array}$ & $\begin{array}{c}41.45 \pm 1.79 \mathrm{e} \\
(62.32)\end{array}$ & $\begin{array}{c}32.45 \pm 1.42 \mathrm{f} \\
(53.09)\end{array}$ \\
\hline $300 \mathrm{mM}$ & $\begin{array}{c}18.76 \pm 1.93 \mathrm{f} \\
(48.96)\end{array}$ & $\begin{array}{c}15.34 \pm 1.84 \mathrm{e} \\
(39.65)\end{array}$ & $\begin{array}{c}27.05 \pm 2.18 \mathrm{~d} \\
(54.81)\end{array}$ & $\begin{array}{c}21.11 \pm 1.89 \mathrm{e} \\
(44.63)\end{array}$ & $\begin{array}{c}33.69 \pm 1.84 \mathrm{e} \\
(57.63)\end{array}$ & $\begin{array}{c}24.34 \pm 1.42 \mathrm{f} \\
(43.63)\end{array}$ & $\begin{array}{l}38.51 \pm 2.49 \mathrm{e} \\
(57.90)\end{array}$ & $\begin{array}{c}27.36 \pm 2.58 \mathrm{~g} \\
(44.76)\end{array}$ \\
\hline $\begin{array}{c}\text { Cultivar } \\
\text { Mean }\end{array}$ & 28.51 & 25.4 & 38 & 33.66 & 45.7 & 39.4 & 51.59 & 42.88 \\
\hline $\mathrm{CV} \%$ & 24.78 & 32.72 & 21.05 & 25.88 & 19.67 & 26.92 & 19.51 & 26.6 \\
\hline
\end{tabular}

* Average value of 9 plants in each treatment.

* Means in a column followed by the same letter do not differ significantly at $5 \%$ level and \pm means standard deviation.

* Values within parenthesis indicate percentage relative to the control. 
Irrigation salinity, growth and yield, Aus rice cultivars, Bangladesh 9

Table 5. Effects of different concentrations of salt ( $\mathrm{NaCl})$ on yield/plant and yield attributes of two rice cultivars BR55 and BR43 at final harvest

\begin{tabular}{|c|c|c|c|c|c|c|c|c|}
\hline \multirow{2}{*}{ Treatments } & \multicolumn{2}{|c|}{ Yield/plant (gm) } & \multicolumn{2}{|c|}{ Panicle no./plant } & \multicolumn{2}{|c|}{ Total grain no./plant } & \multicolumn{2}{|c|}{ Filled grain no./plant } \\
\hline & BR55 & BR43 & BR55 & BR43 & BR55 & BR43 & BR55 & BR43 \\
\hline $0 \mathrm{mM}$ & $6.08 \pm 0.07 \mathrm{a}$ & $4.29 \pm 0.07 \mathrm{a}$ & $6.9 \pm 1.00 \mathrm{a}$ & $6.7 \pm 1.00 \mathrm{a}$ & $551.6 \pm 7.50 \mathrm{a}$ & $388.8 \pm 8.00 \mathrm{a}$ & $390.5 \pm 5.00 \mathrm{a}$ & $275.3 \pm 5.00 \mathrm{a}$ \\
\hline $50 \mathrm{mM}$ & $\begin{array}{c}5.11 \pm 0.05 b \\
(84.04)\end{array}$ & $\begin{array}{c}3.35 \pm 0.07 \mathrm{~b} \\
(78.08)\end{array}$ & $\begin{array}{c}6.6 \pm 1.00 \mathrm{a} \\
(95.65)\end{array}$ & $\begin{array}{c}5.8 \pm 1.00 \mathrm{ab} \\
(86.56)\end{array}$ & $\begin{array}{c}501.7 \pm 6.00 \mathrm{~b} \\
(90.95)\end{array}$ & $\begin{array}{c}341.9 \pm 8.00 \mathrm{~b} \\
(87.93)\end{array}$ & $\begin{array}{c}328.4 \pm 3.00 \mathrm{~b} \\
(84.10)\end{array}$ & $\begin{array}{c}215.2 \pm 5.00 \mathrm{~b} \\
(78.16)\end{array}$ \\
\hline $100 \mathrm{mM}$ & $\begin{array}{c}4.21 \pm 0.06 \mathrm{c} \\
(69.24)\end{array}$ & $\begin{array}{c}2.80 \pm 0.05 \mathrm{c} \\
(65.26)\end{array}$ & $\begin{array}{c}5.7 \pm 1.00 \mathrm{ab} \\
(82.60)\end{array}$ & $\begin{array}{c}5.3 \pm 1.00 \mathrm{abc} \\
(79.10)\end{array}$ & $\begin{array}{c}462.9 \pm 7.00 \mathrm{c} \\
(83.91)\end{array}$ & $\begin{array}{c}326.2 \pm 10.00 \mathrm{bc} \\
(83.89)\end{array}$ & $\begin{array}{c}270.7 \pm 5.00 \mathrm{c} \\
(69.32)\end{array}$ & $\begin{array}{c}180.9 \pm 5.00 \mathrm{c} \\
(65.71)\end{array}$ \\
\hline $150 \mathrm{mM}$ & $\begin{array}{c}3.40 \pm 0.05 \mathrm{~d} \\
(55.92)\end{array}$ & $\begin{array}{c}2.37 \pm 0.07 \mathrm{~d} \\
(55.24)\end{array}$ & $\begin{array}{c}4.6 \pm 1.00 \mathrm{ab} \\
(66.66)\end{array}$ & $\begin{array}{c}4.5 \pm 1.00 \mathrm{abc} \\
(67.16)\end{array}$ & $\begin{array}{c}437 \pm 7.00 \mathrm{~d} \\
(79.22)\end{array}$ & $\begin{array}{c}315.8 \pm 8.00 \mathrm{~cd} \\
(81.22)\end{array}$ & $\begin{array}{c}218.2 \pm 4.00 \mathrm{~d} \\
(55.87)\end{array}$ & $\begin{array}{c}152.6 \pm 5.00 \mathrm{~d} \\
(55.43)\end{array}$ \\
\hline $200 \mathrm{mM}$ & $\begin{array}{l}2.91 \pm 0.05 \mathrm{e} \\
\quad(47.86)\end{array}$ & $\begin{array}{c}1.91 \pm 0.06 \mathrm{e} \\
(44.52)\end{array}$ & $\begin{array}{c}4.1 \pm 1.00 \mathrm{ab} \\
(59.42)\end{array}$ & $\begin{array}{c}4.2 \pm 1.00 \mathrm{abc} \\
(62.68)\end{array}$ & $\begin{array}{c}417.7 \pm 7.00 \mathrm{e} \\
(75.72)\end{array}$ & $\begin{array}{c}306.5 \pm 5.00 \mathrm{cde} \\
(78.83)\end{array}$ & $\begin{array}{c}187.1 \pm 2.00 \mathrm{e} \\
(47.91)\end{array}$ & $\begin{array}{c}123.7 \pm 3.00 \mathrm{e} \\
(44.93)\end{array}$ \\
\hline $250 \mathrm{mM}$ & $\begin{array}{c}2.12 \pm 0.05 \mathrm{f} \\
\quad(34.86)\end{array}$ & $\begin{array}{c}1.51 \pm 0.06 f \\
(35.19)\end{array}$ & $\begin{array}{c}3.5 \pm 1.00 \mathrm{~b} \\
(50.72)\end{array}$ & $\begin{array}{c}3.5 \pm 1.00 \mathrm{bc} \\
(52.23)\end{array}$ & $\begin{array}{c}409.6 \pm 6.00 \mathrm{ef} \\
(72.44)\end{array}$ & $\begin{array}{c}286.1 \mathrm{~d} \pm 6.00 \mathrm{e} \\
(76.15)\end{array}$ & $\begin{array}{c}136.3 \pm 3.00 \mathrm{f} \\
(34.90)\end{array}$ & $\begin{array}{c}97.4 \pm 2.00 \mathrm{f} \\
(35.37)\end{array}$ \\
\hline $300 \mathrm{mM}$ & $\begin{array}{c}2.00 \pm 0.05 \mathrm{~g} \\
(32.89)\end{array}$ & $\begin{array}{c}1.00 \pm 0.06 \mathrm{~g} \\
(23.31)\end{array}$ & $\begin{array}{c}3.1 \pm 1.00 \mathrm{~b} \\
(44.92)\end{array}$ & $\begin{array}{c}2.4 \pm 1.00 \mathrm{c} \\
(35.82)\end{array}$ & $\begin{array}{c}399.8 \pm 5.00 \mathrm{f} \\
(71.42)\end{array}$ & $\begin{array}{c}263.1 \pm 9.00 \mathrm{e} \\
(73.79)\end{array}$ & $\begin{array}{c}117.8 \pm 2.00 \mathrm{~g} \\
(28.88)\end{array}$ & $\begin{array}{c}66.8 \pm 4.00 \mathrm{~g} \\
(27.89) \\
\end{array}$ \\
\hline $\begin{array}{c}\text { Cultivar } \\
\text { Mean }\end{array}$ & 3.65 & 2.48 & 4.92 & 4.62 & 452.07 & 323.17 & 234.85 & 160.27 \\
\hline CV\% & 41.02 & 40.8 & 33.16 & 36.83 & 12.19 & 10.29 & 40.96 & 41.43 \\
\hline
\end{tabular}

* Average value of 9 plants in each treatment.

* Means in a column followed by the same letter do not differ significantly at $5 \%$ level and \pm means standard deviation.

* Values within parenthesis indicate percentage relative to the control. 
Panicle number per plant: Salinity decreased seriously the panicle number per plant (Table 5). At all levels of salinity the absolute panicle number of BR55 was significantly higher than BR43. Compared to control, the highest salinity level significantly reduced panicle number per plant by $55.07 \%$ for BR55 and $64.17 \%$ for BR43. Therefore, significantly higher salt induced reduction of panicle number per plant was observed in BR43 than that in BR55.

Results obtained from this study showed that salinity-induced reduction in the panicle number per plant was mainly attributed to that in the tiller number per plant (Kamal et al., 2015). Similarly, Beatriz et al. (2001) showed that water and soil salinity decrease the number of panicles.

Total grain number per plant: Salinity significantly decreased the total grain number per plant (Table 5). Compared to control, the highest salinity level significantly reduced total grain number per plant by $27.51 \%$ for BR55 and $32.33 \%$ for BR43. Therefore, significantly higher salt induced reduction of total grain number per plant was observed in BR43 than that in BR55. In the present study, the salinity-induced reduction in the grain yield of two rice cultivars resulted from that in the number of panicle per plant (Zeng \& Shannon, 2003).

Filled grain number per plant: Salinity decreased the filled grain number per plant (Table 5).Compared to control, the highest salinity level significantly reduced filled grain number per plant by $69.83 \%$ for BR55 and $75.73 \%$ for BR43.Salinity induced drastically reduction of filled grain number per plant was observed in BR43 than that in BR55. Reduction in filled grain number per plant in this study due to salinity-induced disturbance of source sink relationship of crop plants (Greenway \& Munns, 1980; Blum, 1988). Reduction in grain number per plant in rice have also been reported (Zaibunnisa et al., 2002).

This study indicates that BR55 showed relatively higher tolerance to salinity than BR43 on the basis of growth and yield performance. Therefore, further research should be focused on bio-molecular mechanisms involved in salinity tolerance for the determination of key pathways controlling salinity tolerance in plants.

\section{REFERENCES}

Alamgir, A.N.M. and Ali,M.Y. 2006. Effects of $\mathrm{NaCl}$ salinity on leaf characters and physiological growth attributes of different genotypes of rice (Oryza sativa L.). Bangladesh J. Bot. 35(2): 99-107.

Bal, A.R. and Dutt, S.K. 1984. Effect of soil salinity on growth of Coix lachrymal Jobi L. Ind. J. Plant Physiol., 27: 398-400.

Bangladesh Bureau of Statistics (BBS), 2001. Statistical Year Book of Bangladesh. Statistics Division, Ministry of Planning, Government of the People's Republic of Bangladesh, Dhaka.

Bangladesh Bureauof Statistics (BBS), 2004. Statistical Year Book of Bangladesh. Statistics Division, Ministry of Planning, Government of the People's Republic of Bangladesh, Dhaka. 
Beatriz, G., Piestun, N. and Bernstein, N. 2001. Salinity-induced inhibition of leaf elongation in maize is not mediated by changes in cell wall. Plant Physiol.,125:1419-1428.

Blum, A. 1988. Salinity resistance in Plant Breeding for Stress Environments. CRC Press, Florida. pp. $163-179$.

Cha-um, S. and Kirdmanee, C. 2010. Effect of glycine, betaine and proline on water use and photosynthetic efficiencies and growth of rice seedlings under salt stress. Tur. J. of Agri. andFores.,34: 517-527.

Duan, J., Li, J., Guo, S. and Kang, Y. 2008. Exogenous spermidine affects polyamine metabolism in salinity-stressed Cucumissativus roots and enhances short-term salinity tolerance. $J$. Plant Physiol.,165:1620-1635.

Eugene, V.M., Scott, M.L., Leland, E.F. and Catherine, M.G. 1994. Tiller development in saltstressed wheat. Crop Sci., 34:1594-1603.

FAOSTAT, 2001, Food and Agriculture Organization Corporate Statistical Database, on Agriculture.

Greenway, H. and Munns, R. 1980. Mechanisms of salt tolerance in non-halophytes. Ann. Rev. PL. Physiol.,31: 149-190.

Islam, M.Z., Baset Mia, M.A., Islam, M.R. and Akter, A. 2007. Effect of different saline levels on growth and yield attributes of mutant rice. J. Soil Nature,1(2): 18-22.

Kamal, M.Z.U., Yamaguchi, M., Azuchi F., Kinose, Y., Wada, Y., Funada, R. and Izuta, T. 2015. Effects of ozone and soil salinity, singly and in combination, on growth, yield and leaf gas exchange rates of two Bangladeshi wheat cultivars. Asian J. of Atmos. Env.9-2: 173186.

Karim, Z., Saheed, S.M., Salahuddin, A.B.M., Alam,M.K. and Hoq, A. 1982. Coastal saline soils and their management in Bangladesh. Soil and Irrigation publication No. 8. BARC. Bangladesh.

Mazher, A.M.A., El-Quesni, E.M.F. and Farahat, M.M. 2007. Responses of ornamental and woody trees to salinity. World J. Agric. Sci., 3 (3): 386-395.

Menete, M.Z.L., Van Es, H.M., Brito, R.M.L., DeGloria, S.D. and Famba, S. 2008. Evaluation of system of rice intensification (SRI) component practices and their synergies on saltaffected soils. Field Crop Res., 109:34-44.

Mittler, R., Merquiol, E., Hallak-Herr, E., Rachmilevitch, S., Kaplan, A. and Cohen, M. 2001. Living under a 'dormant' canopy: A molecular acclimation mechanism of the desert plant Retamaraetam. Plant J., 25(4): 407-416.

Netondo, G.W., Onyango, J.C. and Beck, E. 2004. Gas exchange and chlorophyll fluorescence of sorghum under salt stress. Crop Sci. 44:806-811.

Nicolas, M.E., Munns, R., Samarakoon, A.B. and Gifford, R.M. 1994. Elevated $\mathrm{CO}_{2}$ improves the growth of wheat under salinity. Australian. J. Plant Physiol., 20: 349-360.

Saqib, Z.A., Akhtar, J., Ul-Haq,M.A. and Ahmad, I. 2012. Salt induced changes in leaf phenology of wheat plants are regulated by accumulation and distribution pattern of $\mathrm{Na}^{+}$ion. Pak. $J$. Agri. Sci., 49: 141-148.

Siringam, K., Juntawong, N., Cha-um, S. and Kirdmanee, C. 2011. Salt stress induced ion accumulation, ion homeostasis, membrane injury and sugar contents in salt-sensitive rice (Oryza sativa L. spp. indica) roots under isoosmotic conditions. Afr. J. Biotech., 10: 1340-1346.

Soil Resource Development Institute (SRDI), 2010. Saline Soils of Bangladesh. Ministry of Agriculture, Bangladesh: 1-60.

Teixeira, J. and Pereira, S. 2007. High salinity and drought act on an organ-dependent manner on potato glutamine synthetase expression and accumulation. Environ. Exp. Bot.,60:121-126.

Tuna, A.L., Kaya, C., Dikilitas, M. and Higgs, D. 2008. The combined effects of gibberellic acid and salinity on some antioxidant enzyme activities, plant growth parameters and nutritional status in maize plants. Environ. Exp. Bot.,62(1):1-9.

Verma, S. and Mishra, S.N. 2005. Putrescine alleviation of growth in salt stressed Brassica juncea by inducing antioxidative defense system. J. Plant Physiol., 162: 669-677. 
Wankhade, S.D., Cornejo, M.J., Mateu-Andres, I. and Sanz, A. 2013. Morpho-physiological variations in response to $\mathrm{NaCl}$ stress during vegetative and reproductive development of rice. Acta .Physiol. Plant, 35: 323-333.

Weon, Y.C., Kyu, S.L., Jong, C.K., Song, Y.C. and Don, H.C. 2003. Critical saline concentration of soil and water for rice cultivation on a reclaimed saline soil. Korean J. of Crop Sci.,48(3): 238-242.

Zaibunnisa, A., Khan, M.A. and Flowers, T.J. 2002. Causes of sterility in rice under salinity stress. In 'Prospects for Saline Agriculture (Ed. Ahmad, R. and Malik, K.A.). Kluwer Academic Publishers, Netherlands. pp. 177-187.

Zeng, L. and Shannon, M.C. 2000. Salinity effects on seedling growth and yield components of rice. Crop Sci., 40: 996-1003.

Zeng, L. and Shannon, M.C. 2000a. Effects of salinity on grain and yield components of rice at different seeding densities. Agro. J.,92: 418-423.

Zeng, L. and Shannon,M.C. 2003. Salinity Effects on Seedling Growth and Yield Components of Rice.Crop Sci.,40:996-1003.

Zeng, L., Poss, J.A., Wilson, C., Draz, A.E., Gregorio, G.B. and Grieve, C.M. 2003b. Evaluation of salt tolerance in rice genotypes by physiological characters. Euphytica, 129:281-292.

Zhu, J.K. 2001. Plant salt tolerance. Trends Plant Sci., 6(2): 66-71. 